\section{SCIENCE CHINA \\ Physics, Mechanics \& Astronomy}

\title{
Magnetic skyrmion bundles join the family of topological solitons
}

\author{
Nikolai S. Kiselev ${ }^{*}$ \\ Peter Grünberg Institut and Institute for Advanced Simulation, Forschungszentrum Jülich, Jülich D-52425, Germany \\ Received November 26, 2021; accepted December 10, 2021; published online January 28, 2022
}

\begin{tabular}{ll}
\hline Citation: & N. S. Kiselev, Magnetic skyrmion bundles join the family of topological solitons, Sci. China-Phys. Mech. Astron. 65, 247031 (2022), https://doi.org/ \\
& 10.1007/s11433-021-1833-9
\end{tabular}

Magnetic solitons are localized magnetic textures that are similar to ordinary particles - they have defined size and can move and interact with each other. Topological solitons [1], in addition, are characterized by the so-called topological charge or topological index, which allows classifying magnetic solitons and has the following meaning. The two solitons, of different shapes and sizes but identical topological charges, $Q$, can be continuously transformed into each other. On the other hand, such smooth transition does not exist for solitons with different topological indices. The topology of magnetic solitons also contributes to their stability, dynamics, and transport properties. Because of that, the topological magnetic solitons are interesting for fundamental research and hold great promise in developing high-performance spintronic devices [2]. The most promising systems allowing the existence of a wide diversity of such solitons are noncentrosymmetric magnetic crystals with chiral Dzyaloshinsky-Moriya interactions, to which we usually refer simply as chiral magnets.

Nowadays, it is common to name the topological magnetic solitons in these systems as chiral magnetic skyrmions. The magnetic skyrmions were experimentally discovered in these systems more than ten years ago, but up to now, only two types of skyrmions with $Q=-1$ and $Q=0$ have been observed and studied experimentaly [3-5]. This type of solution, known as Bogdanov-Hubert $k \pi$-vortices [6], is characterized by alternating $Q=-1$ and $Q=0$ for odd and even $k$, respectively.
The two independent theoretical studies [7,8] recently suggested a new type of skyrmions solutions named skyrmions bags. Contrary to $k \pi$-vortices, skyrmion bags can have an arbitrary topological index. In the first approximation, skyrmion bags are skyrmions clusters encircled by the closed 180- or 360-degree domain walls. However, it is worth noting that the concept presented in ref. [7] and then developed in ref. [9] provides a more complicated picture with a significantly more rich diversity of skyrmion solutions.

Inspired by these theoretical works on arbitrary topological charge magnetic skyrmions, Tang and coworkers [10] reported an experimental realization of magnetic skyrmions with arbitrary $Q$ in the plate of B20-type FeGe (Figure 1(a)). Furthermore, the supporting numerical simulations provided by the authors revealed that the experimentally observed magnetic texture is characterized by essential inhomogeneities through the plate thickness (Figure 1(b)). Since the three-dimensional magnetic texture has some peculiarities at the top and bottom surfaces of the sample, the authors named this texture skyrmion bundles.

The experimental observation of these solitons became possible due to the well designed experimental setup based on a two-step strategy. First, using a field-cooling process, the mixed state of ordinary $\pi$-skyrmions and helical spin spirals was obtained, and next, the reversed magnetic field was applied. The helical spin spirals surrounding the skyrmions prevent elliptic instability of skyrmions in the inverted magnetic field and finally result in the formation of skyrmion bundles with various topological indices varied in

*Corresponding author (email: n.kiselev@fz-juelich.de) 
(a) Skyrmion

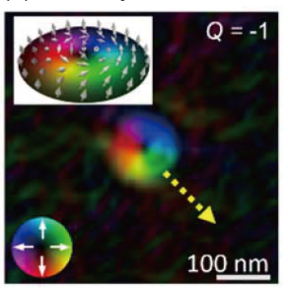

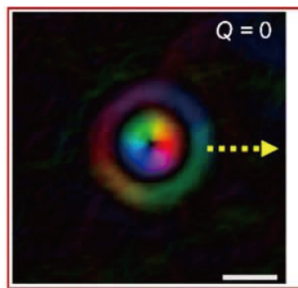

Skyrmion bundle

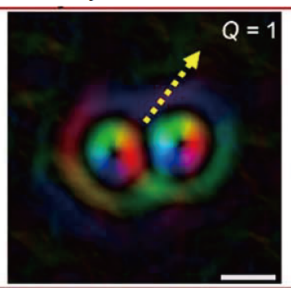

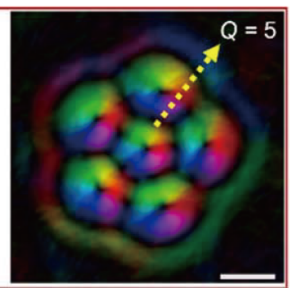

(b)

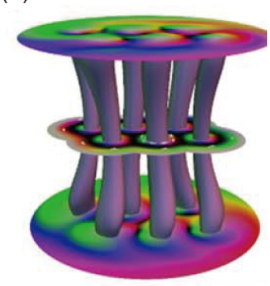

Figure 1 (Color online) (a) Current-driven motion trajectories of skyrmion with $Q=-1$ and skyrmion bundles, with $Q=0$, 1 , and 5. The approximate trajectories of solitons are depicted by yellow arrows and illustrate the dependence of the skyrmion Hall angle on the sign of $Q$. (b) A simulated $3 \mathrm{D}$ magnetic configuration of $Q=5$ skyrmion bundle. This figure is adapted from ref. [10].

a wide range up to $Q=55$. Moreover, the authors also demonstrated the skyrmion bundle motion induced by serials of nanosecond pulsed electric current. It was shown that the dynamics of such diverse skyrmions and, in particular, the deflection angle of skyrmions bundles depends on the topological index of the soliton.

The discovery of skyrmion bundles enriches the family of topological magnetic solitons, provides proof for the theoretical predictions, and could unambiguously promote the development of fundamental research in this field and suggest new concepts for the application of multi- $Q$ solitons in spintronics.

1 N. Manton, and P. Sutcliffe, Topological Solitons (Cambridge University Press, Cambridge, 2004).
2 X. Zhang, Y. Zhou, K. Mee Song, T. E. Park, J. Xia, M. Ezawa, X. Liu, W. Zhao, G. Zhao, and S. Woo, J. Phys.-Condens. Matter 32, 143001 (2020).

3 X. Z. Yu, Y. Onose, N. Kanazawa, J. H. Park, J. H. Han, Y. Matsui, N. Nagaosa, and Y. Tokura, Nature 465, 901 (2010).

4 F. Zheng, H. Li, S. Wang, D. Song, C. Jin, W. Wei, A. Kovács, J. Zang, M. Tian, Y. Zhang, H. Du, and R. E. Dunin-Borkowski, Phys. Rev. Lett. 119, 197205 (2017).

5 J. Tang, L. Kong, Y. Wu, W. Wang, Y. Chen, Y. Wang, J. Li, Y. Soh, Y. Xiong, M. Tian, and H. Du, ACS Nano 14, 10986 (2020).

6 A. Bogdanov, and A. Hubert, J. Magn. Magn. Mater. 195, 182 (1999).

7 F. N. Rybakov, and N. S. Kiselev, Phys. Rev. B 99, 064437 (2019).

8 D. Foster, C. Kind, P. J. Ackerman, J. S. B. Tai, M. R. Dennis, and I. I. Smalyukh, Nat. Phys. 15, 655 (2019).

9 V. M. Kuchkin, B. Barton-Singer, F. N. Rybakov, S. Blügel, B. J. Schroers, and N. S. Kiselev, Phys. Rev. B 102, 144422 (2020).

10 J. Tang, Y. Wu, W. Wang, L. Kong, B. Lv, W. Wei, J. Zang, M. Tian, and H. Du, Nat. Nanotechnol. 16, 1086 (2021). 